\title{
Dysregulation of micro-143-3p and BALBP1 contributes to the pathogenesis of the development of ovarian carcinoma
}

\author{
HONGYAN ZHANG ${ }^{1}$ and WANBIN $\mathrm{LI}^{2}$ \\ ${ }^{1}$ Department of Gynecology, Affiliated Hospital of Jining Medical University, Jining, Shandong 272029; \\ ${ }^{2}$ Jining Medical University, Jining, Shandong 272113, P.R. China
}

Received February 28, 2016; Accepted April 9, 2016

DOI: 10.3892/or.2016.5148

\begin{abstract}
The objective of the present study was to identify the association between mir-143-3p and RalA-binding protein 1 (RALBP1), and their roles in regulating the development of ovarian cancer. Overexpression of RALBP1 induced apoptosis of the ovarian cancer cells, and developed ovarian cancer. In silico analysis and luciferase assay were used to identify whether RALBP1 was the target of mir-143-3p. Subsequently, real-time PCR and western blotting were used to determine the expression level of mir-143-3p, RALBP1 mRNA and protein in different groups, furthermore, MTT assay and flow cytometry were used to detect the viability and apoptosis of cells in different treatment groups. We identified RALBP1 as a target gene of miR-143-3p using computational analysis, and the luciferase activity of cells transfected with wild-type RALBP1 and RALBP1 siRNA were much lower than the scramble control, however, the luciferase activity of cells transfected with mutant RALBP1 was similar with scramble control. The real-time PCR and western blot results suggested that the miR-143-3p level was markedly lower in participants with ovarian cancer compared with normal control, while the expression of RALBP1 mRNA and protein were evidently overexpressed in participants with ovarian cancer compared with normal control. Furthermore, the RALBP1 mRNA and protein level in cells transfected with miR-143-3p mimics and RALBP1 siRNA were downregulated, while notably upregulated subsequent to transfection with miR-143-3p inhibitor, when compared with scramble control. Additionally, the viability of cells were inhibited following transfection with miR-143-3p mimics and RALBP1 siRNA, while notably promoted subsequent to transfection with miR-143-3p inhibitor. Apoptosis of cells were promoted following transfection with miR-143-3p mimics and RALBP1 siRNA, while notably inhibited subsequent to transfection with miR-143-3p
\end{abstract}

Correspondence to: Dr Wanbin Li, Jining Medical University, 16 Taibai Lake Lotus Road, Jining, Shandong 272113, P.R. China E-mail: wbliovaryca@163.com

Key words: micro-143-3p, BALBP1, ovarian carcinoma, tumorigenesis, apoptosis, proliferation inhibitor. These findings provide support that downregulation of the miR-143-3p is associated with a decreased risk of ovarian cancer.

\section{Introduction}

Ovarian cancer is the most life-threatening malignancy in reproductive tract and fourth leading cause of cancer-related deaths in women. Majority of ovarian cancers stem from epithelium, though there are also stromal and germ cell tumors $(1,2)$. Generally, ovarian tumors are silent at early stage and remain unrecognized in most cases $(>80 \%)$ until ovarian carcinoma has metastasized to other areas out of the ovaries. Most recently, 21,550 American new cases of ovarian cancer and 14,600 deaths from the cancer in 2009 were reported by the American Cancer Society (www.cancer.org). There is little improvement in 5-year survival rates over the past 10 years, and long-term survival is significantly unsatisfactory and among the worst of all the anatomic sites of cancer.

Ral paralogs bind to an array of effectors such as ZONAB, phospholipase D1 (PLD1), filamin, exocyst components (Exo84 and Sec5) and RalBP1 (RLIP) upon activation, leading to various functions associated with Ral (3). Earlier findings showed that RalBP1, the best characterized effector of Ral, was correlated with Ral-mediated tumorigenesis (4). Additionally, the human bladder cancer tissues had higher expression of RalBP1 than normal tissues, which was associated with Ral expression (5). The subcutaneous xenograft tumor growth in cancer cell lines could be reduced by deletion of RalBP1 by suppression of its function by antibody or by antisense reduction (6). In RalBP1, there is association between the exocyst complex component Sec5 and transformation mediated by Ral (4). There was also correlation between PLD1 and cell transformation by oncogenes such as Ras (7).

MicroRNAs are non-protein-coding RNAs that play post-transcriptional regulator of genes. microRNAs have been shown to serve a role in almost all cellular processes in animals and plants first discovered as critical regulators of developmental timing in Caenorhabditis elegans $(8,9)$. MicroRNAs have 22 nucleotides, derived from large major transcripts which generate imperfect structures of stem loop. MicroRNAs bind to target mRNAs and suppress transition or enhance transcript degradation, thereby regulating the expression of genes (10). 
Notably, a great deal of microRNAs serve as crucial regulator of gene expression and are generally present in the non-protein-encoding regions (11). More than $60 \%$ of protein-coding transcripts are regulated by small non-coding RNAs known as microRNAs (12). Each microRNA regulates a range of target genes at the post-transcriptional level. They are associated with a variety of disorders and serve as tumor inhibitors and oncogenes to affect tumorigenesis (13). For instance, microRNAs have been associated with ovarian tumor development and progression (14). It is reported that germline variations in processing genes and microRNAs known as messenger RNA transcripts of their target genes play a role in tumor progression as well as the risk of development of cancers, such as ovarian cancer (15).

It has been previously shown that miR-143-3p is differentially expressed in the A2780 cells collected from ovarian carcinoma, and dysregulation of RalA-binding protein 1 (RALBP1) has also been reported to be involved in the molecular mechanism of apoptosis of A2780 cells $(16,17)$. By searching the online miRNA database, we found that RALBP1 is a virtual target of miR-143-3p. In the present study, we validated RALBP1 as a target of miR-143-3p and verified the involvement of miR-143-3p and RALBP1 in the development of ovarian carcinoma.

\section{Materials and methods}

Subjects. The present study consisted of 35 patients treated at the Department of Gynecology, Affiliated Hospital of Jining Medical Unversity (Jining, China) between November 2013 and January 2015. All data processing and sample collection were approved by the Ethics Committee of Affiliated Hospital of Jining Medical Unversity. Participants or their first-degree relatives had already signed the informed consents before start of the experiments after carefully explained all potential risk factors. Cancerous tissue samples and adjacent non-cancerous control were collected from each participant. All of the specimens were obtained from surgical resection. None of the patients was treated with any neoadjuvant treatment, such as radiotherapy and chemotherapy, before surgical resection and the followed tumor resection to collect clinical data.

RNA isolation and real-time PCR. TRIzol reagent (Invitrogen, Carlsbad, CA, USA) was used to extract the total RNA from A2780 cells and tissue samples based on the manufacturer's protocol. NanoDrop spectrophotometer (ND-1000; NanoDrop Technologies, Wilmington, DE, USA) was used to measure the quantity and quality of RNA, and the gel electrophoresis was used to detect the integrity of RNA. For detecting the miR-143-3p expression, miRcute miRNA Isolation kit (Qiangen Biotech Co., Ltd., Beijing, China), miRcute miRNA First-Strand cDNA synthesis kit and miRcute miRNA qPCR detection kit (SYBR-Green) were used to detect the expression of RALBP1 mRNA or miR-143-3p. MiRcute miRNA qPCR detection kit (SYBR-Green) (Qiangen Biotech Co., Ltd.) was used to perform the reverse transcription-polymerase chain reaction (RT-PCR) in accordance with the protocol of the kit supplier with a final $20 \mu \mathrm{l}$ PCR reaction containing $10 \mu \mathrm{l}$ miRcute miRNA premix, $0.4 \mu 1$ reverse primers, $2 \mu 1$ of cDNA samples synthesized and $0.4 \mu 1$ forward. CFX96 ${ }^{\mathrm{TM}}$ Real-Time
System (Bio-Rad, Hercules, CA, USA) was used to carry out the reactions. The internal control was U6. Bio-Rad CFX Manager quantitative PCR software (Bio-Rad) was used to examine the change in expression of miR-143-3p and RALBP1 mRNA, and the $2^{-\Delta \Delta C t}$ method was used to calculate the change in expression of RALBP1 gene and miR-143-3p. Three independent tests were performed.

Cell culture and transfection. Dulbecco's modified Eagle's medium (DMEM) (Cellgro Mediatech, Manassas, VA, USA) containing $1 \%$ non-essential amino acids (Sigma-Aldrich, St. Louis, MO, USA), $2 \mathrm{mM}$ L-glutamine, $50 \mu \mathrm{g} / \mathrm{ml}$ streptomycin sulfate, $50 \mathrm{U} / \mathrm{ml}$ penicillin and $10 \%$ fetal bovine serum (FBS) (Invitrogen) was used to maintain the A2780 cells in an atmosphere of $5 \% \mathrm{CO}_{2} / 95 \%$ air at $37^{\circ} \mathrm{C}$. When cells were cultured to $80 \%$ confluency, transfection was performed using Lipofectamine 2000 (Invitrogen) according to the manufacturer's protocol. A2780 cells were analyzed $48 \mathrm{~h}$ after transfection.

Cell proliferation assay. A2780 cell proliferation was measured using the Alamar Blue assay according to the quantitative metabolic conversion of non-fluorescent resazurin and blue to fluorescent resorufin and pink by living A2780 cells. An Alamar Blue (Invitrogen) stock solution was added to the wells equal to $10 \%$ of the total incubation volume for 2-6 h. Synergy HT Multi-Mode Microplate Reader (Bio-Tek Instruments, Winooski, VT, USA) was used to determine the reduction of resazurin in the cultures according to the absorbance at 590 and $530 \mathrm{~nm}$ wavelengths. Each test was performed at least three times.

Luciferase assay. The PCR fragment including the binding site was used to make the luciferase constructs. The 3' untranslated region (3'UTR) of RALBP1 with the target site of miR-143-3p was inserted into the XhoI and NotI sites of the psiCHECK-2 (Promega, Madison, WI, USA) following the manufacturer's recommendations. Mutations were introduced using site-directed mutagenesis (Stratagene, La Jolla, CA, USA). For the reporter assays, Lipofectamine 2000 was used to co-transfect A2780 cells with negative control (NC mimics), mutant miR-143-3p or wild-type miR-143-3p mimics (SunBio Corporation, Shanghai, China) and reporter plasmids (500 ng). Promega Luciferase Assay System was used to determine luciferase activity according to the activity of Renilla luciferase (control). All tests were repeated in triplicate.

Western blot analysis. For analysis of the expression of RALBP1 protein, the tissue sample or culture cells were lyzed using lysis buffer including $1 \mathrm{mmol} / \mathrm{l}$ sodium orthovanadate, $2 \mathrm{mmol} / \mathrm{l}$ EDTA, $150 \mathrm{mmol} / \mathrm{l} \mathrm{NaCl}, 25 \mu \mathrm{g} / \mathrm{ml}$ leupeptin, $1 \%$ deoxycholate plus, $10 \mu \mathrm{g} / \mathrm{ml}$ aprotinin, $1 \%$ Triton X-100 and $50 \mathrm{mmol} / 1$ Tris-HCl $\mathrm{pH}$ 7.4. Then, the cellular lysis were centrifuged for $15 \mathrm{~min}$ at $15,000 \mathrm{rpm}$, and the Bradford assay (Bio-Rad) was used to analyze the quantity of the cellular protein. SDS-PAGE (12\%) was used to separate the lysates, and then electro-transferred onto polyvinylidene difluoride (PVDF) membranes (PerkinElmer, Waltham, MA, USA) for $2 \mathrm{~h}(90 \mathrm{~V})$. Tris-buffered saline containing $0.1 \%$ Tween-20 (TBST) with 


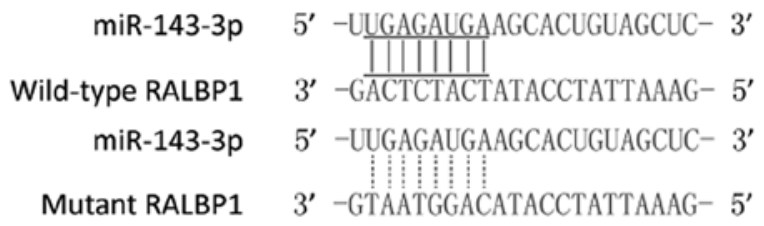

Figure 1. The 3'-UTR of RALBP1 is targeted by miR-143-3p with potential 'hits' in the 3'UTR of RALBP1.

$5 \%$ non-fat dry milk (avoid non-specificity binding) was used to block the membranes for $2 \mathrm{~h}$. Then, monoclonal antibodies anti-human RALBP1 (1:1,000; Santa Cruz Biotechnology, Shanghai, China) were used to incubate the membranes for $12 \mathrm{~h}$ at $4^{\circ} \mathrm{C}$ in accordance with the manufacturer's description, then the membranes were washed three times using PBST (BioSharp, Hefei, China); next, secondary antibody at 1:5,000 dilution (Cell Signaling Technologies, Cambridge, MA, USA) in PBST was used to treat the membranes for another $1 \mathrm{~h}$, subsequently the membranes were washed three times using PBST for $5 \mathrm{~min}$. The enhanced chemiluminescence (ECL) detection system (Pierce Biotechnology, Inc., Rockford, IL, USA) was used to develop the bands following the manufacturer's protocol. Each test was repeated in triplicate.

Apoptosis analysis. Annexin V/propidium iodide staining with the apoptosis detection kit (KenGEN, China) was used to detect A2780 cell apoptosis based on the manufacturer's protocol. Annexin V was used to stain the A2780 cells subsequently, and then FACSCalibur flow cytometer (BD Biosciences, New Jersey, NY, USA) was used to analyze cell apoptosis. Each test was repeated in triplicate.

Statistical analysis. All data are presented as mean \pm SD (standard deviation). Every experiment was analyzed three times, with three samples for each. Student's t-test was used to evaluate the comparisons of treatment group. p-value $<0.05$ was considered to indicate a statistically significant result. Each test was repeated in triplicate.

\section{Results}

RALBP1 is a target of miR-143-3p. The relationship between the RALBP1-3'UTR and its targeted miRNAs was predicted through bioinformatics analysis by the bioinformatics algorithms TargetScan http://www.targetscan. org/) and miRanda (http://www.microrna.org/microrna/ home.do) which showed that the 3'-UTR of RALBP1 may be targeted by miR-143-3p with potential 'hits' in the 3'UTR of RALBP1 (Fig. 1).

Luciferase assay was used to further confirm the association between miR-143-3p and RALBP1. Then, the cells were transfected with construct including wild-type or mutant RALBP1 3'UTR. As shown in Fig. 2, we found that the luciferase activity of the cells co-transfected with miR-143-3p mimics and wildtype RALBP1 3'UTR was lower than those transfected with the scramble controls, while the introduction of mutant RALBP1 with the potential 'seed sequence' in the 3'TUR of RALBP1 almost completely abolished such repressive effect, indicating that miR-143-3p negatively regulated RALBP1.

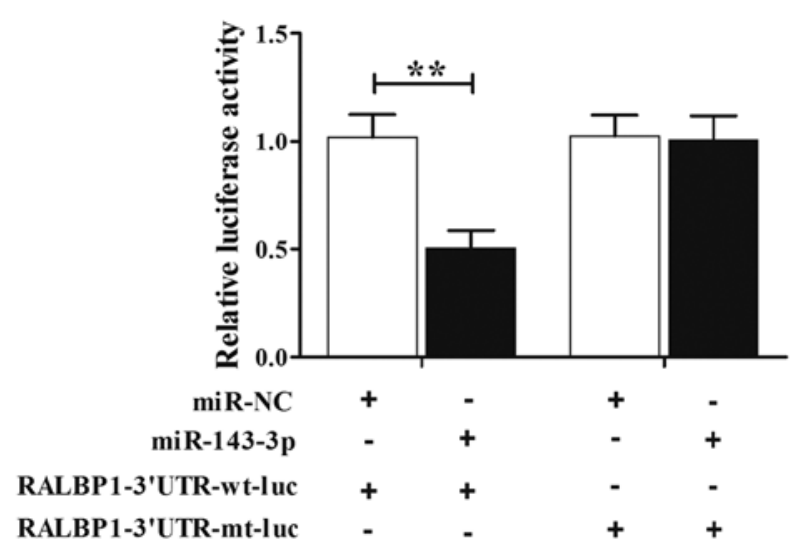

Figure 2. Luciferase assay showed that the miR-143-3p mimics overexpressing A2780 cells transfected with wild-type RALBP1 had a significantly lower luciferase activity compared with those cells transfected with mutant RALBP1 and scramble control

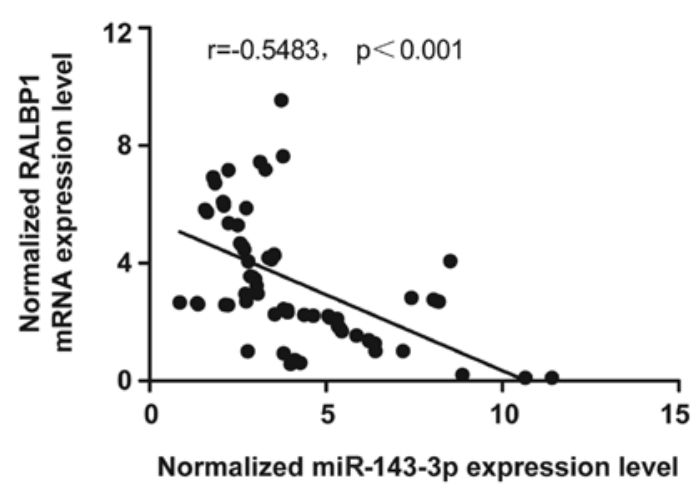

Figure 3. The expression level of RALBP1 mRNA and the expression of miR-143-3p were measured using real-time PCR to validate miRNA-mRNA regulatory relationship. We confirmed the negative regulatory relationship between miR-143-3p and RALBP1, and the negative correlation coefficient was $-0.5483(r=-0.5483)$.

The negative regulatory relationship between $R A L B P 1$ and $m i R-143-3 p$. The miRNA-mRNA regulatory relationship was confirmed using RT-PCR. The results confirmed the negative regulatory relationship between miR-143-3p and RALBP1, and the negative correlation coefficient was -0.5483 ( $\mathrm{r}=-0.5483)$, as shown in Fig. 3.

Upregulation of $m i R-143-3 p$ reduces the expression of $R A L B P 1$. Using real-time PCR and western blot analyses, we found the expression of miR-143-3p (Fig. 4A) was much lower in participants with compared with normal control, while the expression of RALBP1 mRNA (Fig. 4B) and protein (Fig. 4C) were evidently overexpressed in participants with ovarian cancer compared with normal control, indicated that downregulated expression of $\mathrm{miR}-143-3 \mathrm{p}$ and overexpression of RALBP1 can induce the development of ovarian cancer.

Effect of miR-143-3p on the expression of RALBP1. To further confirm whether RALBP1 negatively correlated with miR-143-3p, we investigated the mRNA and protein expression level of RALBP1 in A2780 cells. As shown in Fig. 5, the expression level of RALBP1 mRNA (Fig. 5A) and protein (Fig. 5B) in 
A

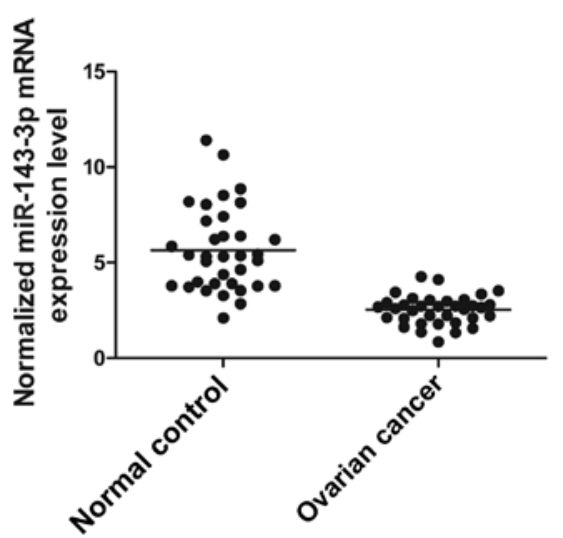

B

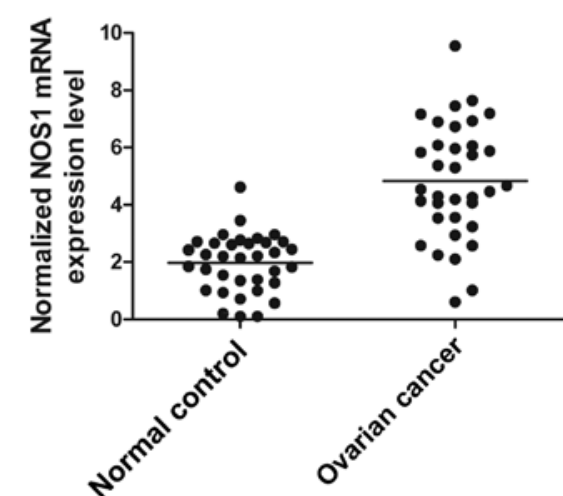

C

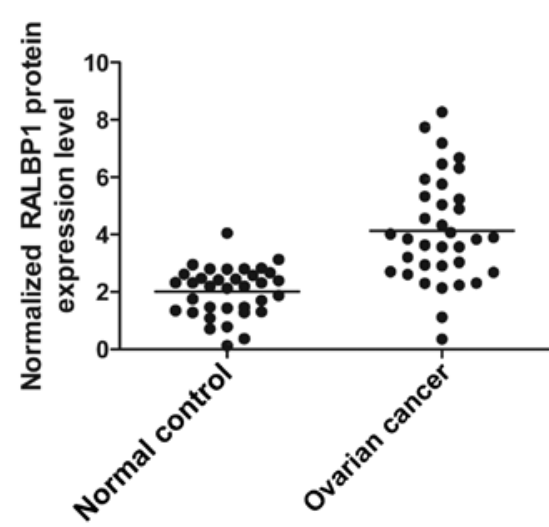

Figure 4. Real-time PCR and western blot analyses were used to detect the expression of (A) miR-143-3p, (B) RALBP1 mRNA and (C) protein in different groups, and the expression of miR-143-3p (A) was much lower, while the expression of RALBP1 mRNA (B) and protein (C) were evidently overexpressed in participants with ovarian cancer compared with normal control.

cells transfected with miR-143-3p mimics or RALBP1 siRNA was significantly lower than in the scramble control, while the RALBP1 mRNA (Fig. 5A) and protein (Fig. 5B) level were evidently upregulated following transfection with miR-143-3p inhibitor. These observations indicated that there was negative regulatory relationship between miR-143-3p and RALBP1.

Introduction of miR-143-3p significantly affects the viability and apoptosis in A2780 cells. To study the molecular mechanism underlying the observed viability-reduced and apoptosis-promoting effect of miR-143-3p, we performed flow cytometry analysis in the cells transfected with miR-143-3p mimics, miR-143-3p inhibitor and RALBP1 siRNA, as shown in Fig. 6A, the viability of cells transfected with miR-143-3p mimics or RALBP1 siRNA was evidently reduced compared with scramble control, while the viability of cells transfected with miR-143-3p inhibitor was markedly higher than scramble control. As shown in Fig. 6B, apoptosis of cells transfected with miR-143-3p mimics or RALBP1 siRNA was evidently promoted compared with scramble control, while apoptosis of cells transfected with miR-143-3p inhibitor was markedly reduced compare to scramble control. These data indicated the viability-reduced and apoptosis-promoting effect of miR143-3p.

\section{Discussion}

We discovered that a miRNA cluster known as miR-145-5p/ miR-143-3p may have positive regulatory role in the cell proliferation of CIK cells using chromosome clustering and GO analysis. Notably, this miRNA cluster has been predicted to target proto-oncogene such as c-Myc, Bcl-2 and Ras family. Nevertheless, we only observed significant upregulation of c-Myc and Bcl-2 during CIK production. During CIK preparation, the two genes that are anti-apoptotic may be implicated in the proliferation of cells (18). It has been shown that the downregulated expression levels of miR-143-3p and miR-143-5p were observed in gastric cancer, which complied with results described by other study teams $(19,20)$. Recently, investigators analyzed 70 paired samples of benign tissues and gastric cancers using real-time RT-PCR and chip assays (20). They discovered greatest downregulation of miR-143 among miRNAs in gastric cancers, when compared with benign tissues. There was correlation between the expression level of miR-143 and the progression of gastric cancer and stage IV cancers had significantly lower level of miR-143 than stage I and II cancers. Takagi et al found downregulation of miR-143 in gastric cancer cell lines and that the viability of gastric cancer cells was suppressed by transfection with miR-143-3p which targeted ERK5 and AKT consistent with our observations (19). Nevertheless, the role of miR-143-5p in gastric cancer remains unknown. In the present study, computational analysis [bioinformatics algorithms TargetScan (http://www.targetscan.org/) and miRanda (http://www.microrna.org/microrna/home.do)] was used to identify that RALBP1 was a target gene of miR-143-3p, and further confirmed using luciferase assay, the results shown that luciferase activity of the cells co-transfected with wild-type RALBP1 3'UTR was lower than those transfected with the scramble controls, while the introduction of mutant RALBP1 with the potential 'seed sequence' in the 3'TUR of RALBP1 almost completely abolished such repressive effect. The miR-143-3p level was determined using western blotting and real-time PCR, we found that the expression of miR-143-3p mRNA was markedly downregulated in participants with ovarian cancer compared with normal control, while the expression of RALBP1 mRNA and protein were evidently overexpressed in participants with ovarian cancer compared with normal control.

RLIP76 has been reported to be a novel R-Ras effector linking R-Ras to the activation of Arf6 and subsequently, to Rac1 resulting in cell migration and spreading dependent on adhesion. Additionally, the various roles of RLIP76 may contribute to multiple functions of R-Ras. For instance, studies 
A

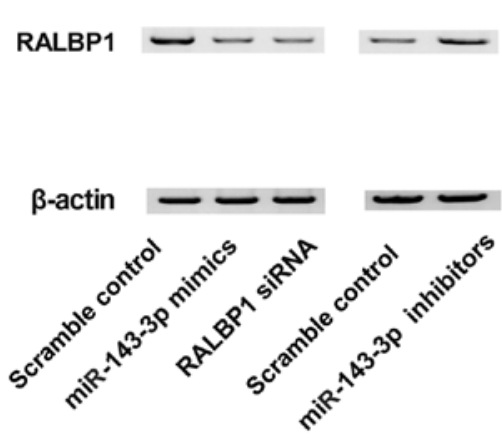

B

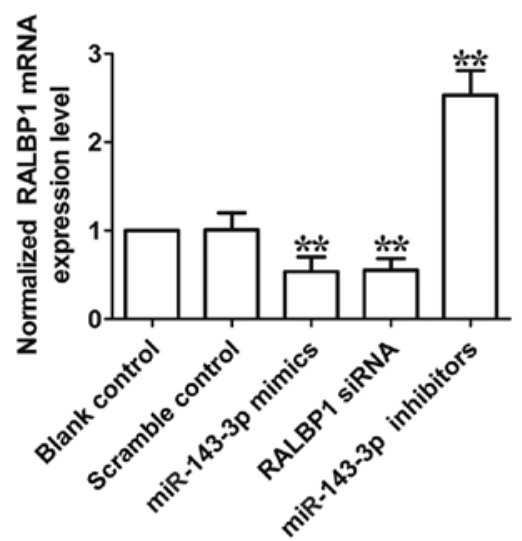

Figure 5. The impacts the miR-143-3p on the expression of the RALBP1 mRNA and protein were estimated using real-time PCR and western blotting, and the expression level of (A) RALBP1 mRNA and (B) protein in cells transfected with miR-143-3p mimics or RALBP1 siRNA was significantly lower than the scramble control, while the RALBP1 mRNA (A) and protein (B) level were evidently upregulated following transfection with miR-143-3p inhibitor.

A

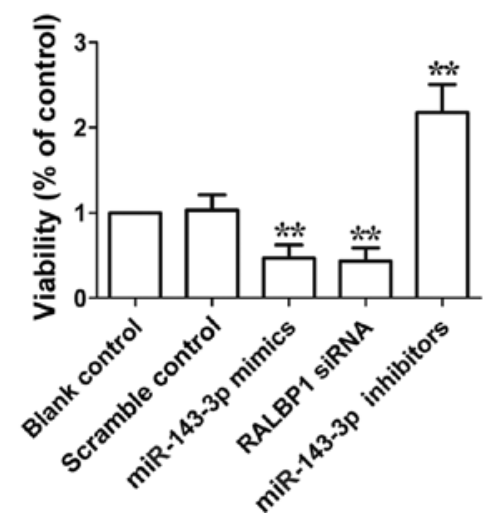

B

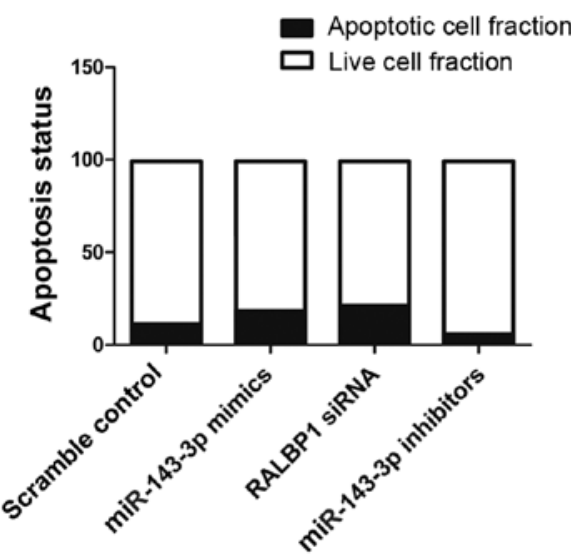

Figure 6. Introduction of miR-143-3p mimics or RALBP1 siRNA significantly suppressed the viability of (A) A2780 cells, while introduction of miR-143-3p inhibitor significantly promoted compared with the scramble control. As shown in B, the apoptosis of cells transfected with miR-143-3p mimics or RALBP1 siRNA was evidently promoted, while the apoptosis of cells transfected with miR-143-3p inhibitor was markedly reduced compared to scramble control.

of Keely et al in 1999, and Holly et al in 2005, showed that the activation of Rac GTPase dependent on RLIP76 may account for the function of R-Ras to enhance neurite outgrowth and cell migration, which were Rac-dependent processes $(21,22)$. Moreover, in 1997, Radhakrishna and Donaldson demonstrated that the adhesion-induced active Arf6 GTPase, known as a regulator of vesicle trafficking, was triggered by RLIP76 (23); therefore, regulation of Arf6 may account for the function of RLIP76 to regulate endocytosis (24). In fact, there is physical correlation between RLIP76 and $\mu 2$ chain of AP-2, an adaptor with membrane recruitment regulated by Arf6, demonstrating that endocytosis mediated by clathrin can be coordinately regulated by the two proteins clathrinmediated (25). Their function to facilitate cell proliferation can be limited by endocytosis of growth factor receptors; hence, the ability of R-Ras limiting the proliferation of smooth muscle cells and endothelial cells can be explained by the interaction of RLIP76 with R-Ras (26).

Molecules that regulate signaling networks of angiogenesis are potential therapeutic targets. Ral-interacting protein of
$76 \mathrm{kDa}$ (RLIP76, also RalBP1) has been identified as a potential target, contributing to its physiological and cellular properties that are still being studied, and to the substantial reduction in tumors obtained by blockade of RLIP76 in numerous tumor models (reviewed in ref. 27). As a pleiotropic protein, RLIP76 emerged as a Ral GTPase effector protein connecting Ral to Rho pathways via its activity of RhoGAP (28). In addition, RLIP76 serves as an ATP-dependent glutathione-conjugated transporter for small molecules, such as endogenous metabolites and anticancer drugs, and in cell spreading and migration, mitochondrial fission and endocytosis (29-32). A number of signaling molecules can bind to the sites of the protein (28); therefore, RLIP76 seems to back up a regulatory scaffolding function for signaling. Majority of human tissues such as kidney, muscle, lung, ovary, heart and liver, and majority of human tumor cell lines can express RLIP76, and overexpression of RLIP76 is observed in many cancers including melanomas, ovarian carcinomas and lung cancer (27). There is a correlation between blockade of RLIP76 with antisense or targeting antibodies and enhanced sensitivity to chemotherapy 
and radiotherapy, which results in substantial tumor regression in B16 melanomas, prostate cancer, colon and non-small cell lung carcinomas in mice $(6,33,34)$. Nevertheless, tumor regression observed in the studies may have been obtained from effects in either the animal host cells or the tumor cells, or both. Therefore, RLIP76 is necessary for cancer survival and progression although the mechanisms are unknown. In the present study, the RALBP1 mRNA and protein level in cells transfected with miR-143-3p mimics and RALBP1 siRNA were downregulated, while notably upregulated subsequent to transfection with miR-143-3p inhibitor, when compared with scramble control. Additionally, flow cytometric analysis was used to study the molecular mechanism underlying the observed viability-reduced and apoptosis-promoting effect of miR-143-3p, the results suggested the viability of cells was inhibited following transfection with miR-143-3p mimics and RALBP1 siRNA, while notably promoted subsequent to transfection with miR-143-3p inhibitor. Apoptosis of cells were promoted following transfection with miR-143-3p mimics and RALBP1 siRNA, while notably inhibited subsequent to transfection with miR-143-3p inhibitor. In conclusion, these findings provide support that downregulation of miR-143-3p caused an increased expression of RALBP1, which may be a molecular mechanism of tumorigenesis of ovarian cancer. miR-143-3p and RALBP1 show promise as therapeutic targets in the management of ovarian cancer.

\section{References}

1. Parkin DM, Bray F, Ferlay J and Pisani P: Estimating the world cancer burden: Globocan 2000. Int J Cancer 94: 153-156, 2001.

2. Kaku T, Ogawa S, Kawano Y, Ohishi Y, Kobayashi H, Hirakawa T and Nakano H: Histological classification of ovarian cancer. Med Electron Microsc 36: 9-17, 2003.

3. Bodemann BO and White MA: Ral GTPases and cancer: Linchpin support of the tumorigenic platform. Nat Rev Cancer 8: 133-140, 2008.

4. Lim KH, Baines AT, Fiordalisi JJ, Shipitsin M, Feig LA, Cox AD, Der CJ and Counter CM: Activation of RalA is critical for Ras-induced tumorigenesis of human cells. Cancer Cell 7: 533-545, 2005.

5. Smith SC, Oxford G, Baras AS, Owens C, Havaleshko D, Brautigan DL, Safo MK and Theodorescu D: Expression of ral GTPases, their effectors, and activators in human bladder cancer. Clin Cancer Res 13: 3803-3813, 2007.

6. Singhal SS, Awasthi YC and Awasthi S: Regression of melanoma in a murine model by RLIP76 depletion. Cancer Res 66: 2354-2360, 2006.

7. Jiang H, Lu Z, Luo JQ, Wolfman A and Foster DA: Ras mediates the activation of phospholipase D by v-Src. J Biol Chem 270: 6006-6009, 1995.

8. He L and Hannon GJ: MicroRNAs: Small RNAs with a big role in gene regulation. Nat Rev Genet 5: 522-531, 2004.

9. Reinhart BJ, Weinstein EG, Rhoades MW, Bartel B and Bartel DP: MicroRNAs in plants. Genes Dev 16: 1616-1626, 2002.

10. Bartel DP: MicroRNAs: Target recognition and regulatory functions. Cell 136: 215-233, 2009.

11. Kim VN and Nam JW: Genomics of microRNA. Trends Genet 22: 165-173, 2006.

12. Esteller M: Non-coding RNAs in human disease. Nat Rev Genet 12: 861-874, 2011.

13. Esquela-Kerscher A and Slack FJ: Oncomirs - microRNAs with a role in cancer. Nat Rev Cancer 6: 259-269, 2006.

14. Shahab SW, Matyunina LV, Mezencev R, Walker LD, Bowen NJ, Benigno BB and McDonald JF: Evidence for the complexity of microRNA-mediated regulation in ovarian cancer: A systems approach. PLoS One 6: e22508, 2011.
15. Permuth-Wey J, Kim D, Tsai YY, Lin HY, Chen YA, Barnholtz-Sloan J, Birrer MJ, Bloom G, Chanock SJ, Chen Z, et al; Ovarian Cancer Association Consortium: LIN28B polymorphisms influence susceptibility to epithelial ovarian cancer. Cancer Res 71: 3896-3903, 2011.

16. Nam EJ, Yoon H, Kim SW, Kim H, Kim YT, Kim JH, Kim JW and Kim S: MicroRNA expression profiles in serous ovarian carcinoma. Clin Cancer Res 14: 2690-2695, 2008.

17. Hudson ME, Pozdnyakova I, Haines K, Mor G and Snyder M: Identification of differentially expressed proteins in ovarian cancer using high-density protein microarrays. Proc Natl Acad Sci USA 104: 17494-17499, 2007.

18. Nakagawa M, Tsuzuki S, Honma $\mathrm{K}$, Taguchi $\mathrm{O}$ and Seto $\mathrm{M}$ : Synergistic effect of $B c l 2, M y c$ and $C c n d l$ transforms mouse primary B cells into malignant cells. Haematologica 96: 1318-1326, 2011.

19. Takagi T, Iio A, Nakagawa Y, Naoe T, Tanigawa N and Akao Y: Decreased expression of microRNA-143 and -145 in human gastric cancers. Oncology 77: 12-21, 2009.

20. Li X, Luo F, LiQ, Xu M, Feng D, Zhang G and Wu W: Identification of new aberrantly expressed miRNAs in intestinal-type gastric cancer and its clinical significance. Oncol Rep 26: 1431-1439, 2011.

21. Keely PJ, Rusyn EV, Cox AD and Parise LV: R-Ras signals through specific integrin alpha cytoplasmic domains to promote migration and invasion of breast epithelial cells. J Cell Biol 145: 1077-1088, 1999.

22. Holly SP, Larson MK and Parise LV: The unique N-terminus of $\mathrm{R}$-ras is required for Rac activation and precise regulation of cell migration. Mol Biol Cell 16: 2458-2469, 2005.

23. Radhakrishna $\mathrm{H}$ and Donaldson JG: ADP-ribosylation factor 6 regulates a novel plasma membrane recycling pathway. J Cell Biol 139: 49-61, 1997.

24. Jullien-Flores V, Mahé Y, Mirey G, Leprince C, MeunierBisceuil B, Sorkin A and Camonis JH: RLIP76, an effector of the GTPase Ral, interacts with the AP2 complex: Involvement of the Ral pathway in receptor endocytosis. J Cell Sci 113: 2837-2844, 2000.

25. Paleotti O, Macia E, Luton F, Klein S, Partisani M, Chardin P, Kirchhausen T and Franco M: The small G-protein Arf6GTP recruits the AP-2 adaptor complex to membranes. J Biol Chem 280: 21661-21666, 2005.

26. Ceresa BP and Schmid SL: Regulation of signal transduction by endocytosis. Curr Opin Cell Biol 12: 204-210, 2000.

27. Awasthi S, Singhal SS, Awasthi YC, Martin B, Woo JH, Cunningham CC and Frankel AE: RLIP76 and cancer. Clin Cancer Res 14: 4372-4377, 2008.

28. Jullien-Flores V, Dorseuil O, Romero F, Letourneur F, Saragosti S, Berger R, Tavitian A, Gacon G and Camonis JH: Bridging Ral GTPase to Rho pathways. RLIP76, a Ral effector with CDC42/Rac GTPase-activating protein activity. J Biol Chem 270: 22473-22477, 1995.

29. Awasthi S, Cheng JZ, Singhal SS, Pandya U, Sharma R, Singh SV, Zimniak P and Awasthi YC: Functional reassembly of ATP-dependent xenobiotic transport by the N- and C-terminal domains of RLIP76 and identification of ATP binding sequences. Biochemistry 40: 4159-4168, 2001.

30. Goldfinger LE, Ptak C, Jeffery ED, Shabanowitz J, Hunt DF and Ginsberg MH: RLIP76 (RalBP1) is an R-Ras effector that mediates adhesion-dependent Rac activation and cell migration. J Cell Biol 174: 877-888, 2006.

31. Kashatus DF, Lim KH, Brady DC, Pershing NL, Cox AD and Counter CM: RALA and RALBP1 regulate mitochondrial fission at mitosis. Nat Cell Biol 13: 1108-1115, 2011.

32. Nakashima S, Morinaka K, Koyama S, Ikeda M, Kishida M, Okawa K, Iwamatsu A, Kishida S and Kikuchi A: Small G protein Ral and its downstream molecules regulate endocytosis of EGF and insulin receptors. EMBO J 18: 3629-3642, 1999.

33. Singhal SS, Roth C, Leake K, Singhal J, Yadav S and Awasthi S: Regression of prostate cancer xenografts by RLIP76 depletion. Biochem Pharmacol 77: 1074-1083, 2009.

34. Singhal SS, Singhal J, Yadav S, Dwivedi S, Boor PJ, Awasthi YC and Awasthi S: Regression of lung and colon cancer xenografts by depleting or inhibiting RLIP76 (Ral-binding protein 1). Cancer Res 67: 4382-4389, 2007. 